$10-1-2020$

\title{
Orientation des programmes pour le changement social et de comportement à travers l'écoute sociale et le suivi social
}

Breakthrough RESEARCH

Follow this and additional works at: https://knowledgecommons.popcouncil.org/departments_sbsr-pgy

Part of the Health Communication Commons, and the Public Health Commons How does access to this work benefit you? Let us know!

\section{Recommended Citation}

Breakthrough RESEARCH. 2020. "Orientation des programmes pour le changement social et de comportement à travers l'écoute sociale et le suivi social," Breakthrough RESEARCH Note de recherche programmatique. Washington, DC: Population Council. 


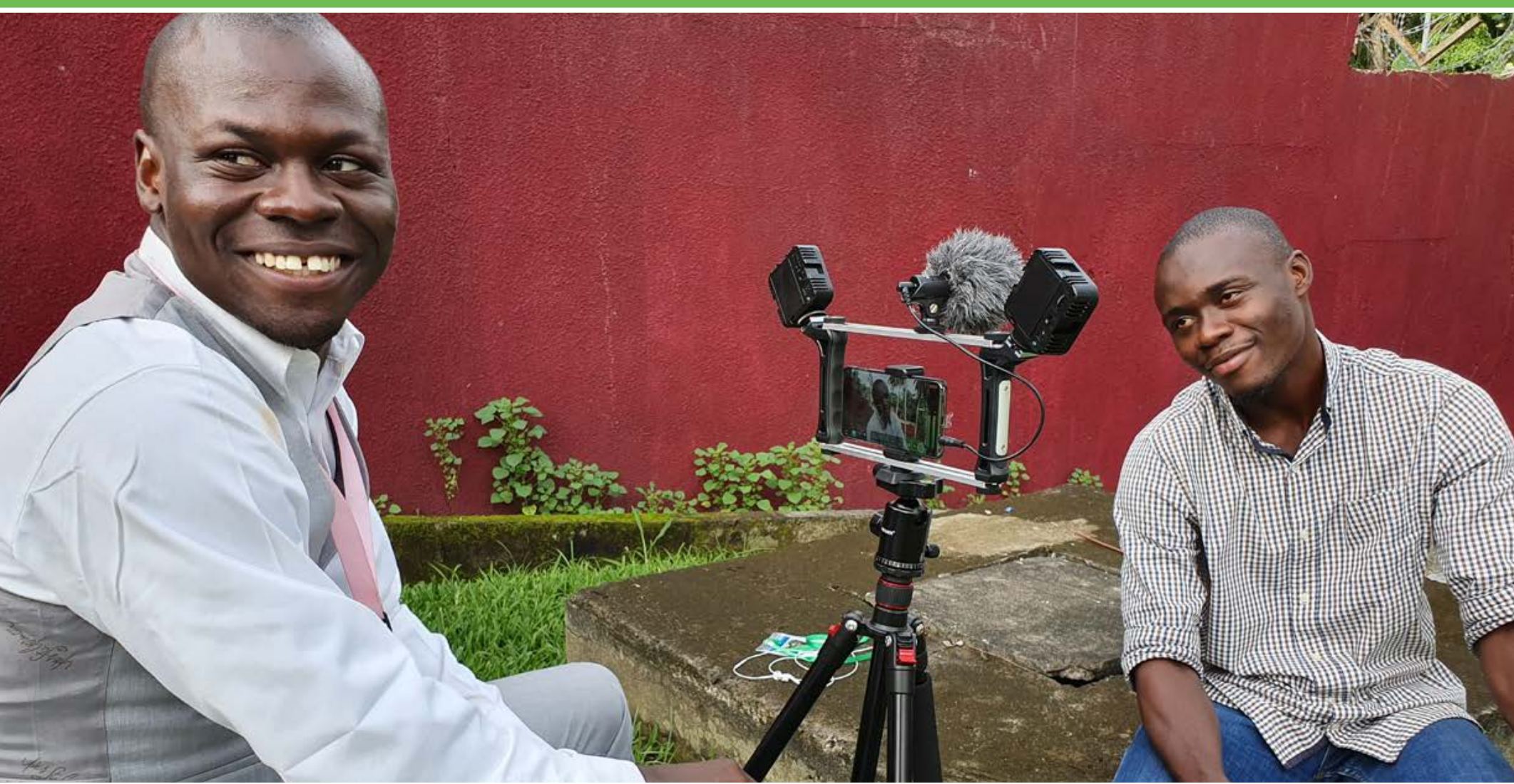

\section{Orientation des programmes pour le changement social et de comportement} À TRAVERS L'ÉCOUTE SOCIALE ET LE SUIVI SOCIAL

Cette note de recherche offre des conseils pratiques quant à la manière d'utiliser l'écoute sociale et le suivi social comme outils permettant d'orienter les programmes de changement social et de comportement (CSC). Elle est destinée aux agents de mise en œuvre des programmes CSC au niveau mondial et régional, aux évaluateurs et aux bailleurs de fonds dans les pays prioritaires soutenus par l'USAID. Cette note donne un aperçu de l'écoute sociale et du suivi social et explique comment ces méthodes peuvent constituer des outils importants dans la collecte de renseignements concernant les connaissances et les attitudes de publics cibles, ainsi que leur exposition et leurs réponses à des interventions CSC particulières. À l'aide d'exemples appliqués en Afrique de l'Ouest francophone, elle illustre les étapes entreprises dans l'écoute sociale et le suivi social, met en avant certains enseignements tirés de ces approches et détermine comment ces méthodes peuvent être appliquées aux programmes CSC. 


\section{MESSAGES CLÉS}

- Les personnes dans le monde entier utilisent de plus en plus les réseaux sociaux pour s'exprimer et initier des conversations concernant leur vie et leur santé.

- Les programmes de changement social et de comportement peuvent exploiter l'écoute sociale pour obtenir des renseignements liés aux connaissances, attitudes et comportements des personnes sur leur santé.

- Les renseignements générés par le biais de l'écoute sociale et du suivi des réseaux sociaux peuvent être utilisés conjointement avec d'autres techniques de suivi et d'évaluation pour produire des environnements d'apprentissage riches en données, en vue de concevoir et d'adapter des programmes.

\section{Contexte}

Les personnes dans le monde entier utilisent de plus en plus les réseaux sociaux pour s'exprimer et initier des conversations concernant leur vie et leur santé. En 2020, près de trois milliards de personnes utilisaient les réseaux sociaux (voir Figure 1). ${ }^{1}$ Les réseaux sociaux renvoient aux sites web et aux applications permettant aux utilisateurs de créer et de partager du contenu ou de prendre part à des réseaux sociaux en ligne. Parmi les exemples de ces réseaux figurent Facebook, Instagram, Twitter, YouTube, Reddit, des blogs, des sites d'informations, etc. ${ }^{2}$ À travers l'écoute sociale et le suivi social, leurs conversations et commentaires peuvent fournir quantité de renseignements aux chercheurs et aux agents chargés de la planification des programmes.

L'écoute sociale désigne le processus consistant à suivre le nombre de mentions et le contenu de conversations liés à un sujet, à un programme ou à une marque sur un réseau social. ${ }^{3}$ Pour les projets CSC, l'écoute sociale peut constituer un outil important afin de comprendre les croyances, les attitudes et les comportements. L'écoute sociale permet aux programmes CSC de noter ce qui est dit sur les réseaux sociaux, d'analyser le contenu et le sentiment derrière les messages, notamment les informations erronées, et d'utiliser ces renseignements pour concevoir des programmes et adapter les modes de gestion.

Le suivi social, également désigné comme suivi des réseaux sociaux, est lié à l'écoute sociale et peut servir à cibler l'engagement du public et ses réactions face à certains messages partagés en rapport avec un produit ou un programme particulier. Les changements de suivi en termes d'engagement et de réponses permettent aux gestionnaires des programmes de mesurer les publics atteints et leur opinion quant aux conversations. ${ }^{4}$

\section{FIGURE 1. LES UTILISATEURS DES RÉSEAUX SOCIAUX ONT FRANCHI LA BARRE DES 3 MILLIARDS EN 2020}

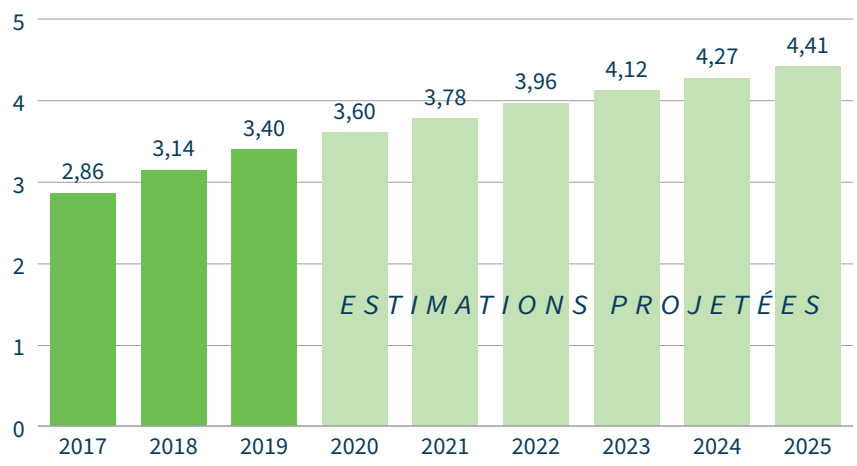

Source : Statista, Dossier: Social Media Usage Worldwide (2020),

https://www.statista.com/study/12393/social-networks-statista-dossier/.

Ces méthodes sont de plus en plus pertinentes pour le travail dans le domaine du CSC au sein des pays prioritaires de l'Agence des États-Unis pour le développement international (USAID), dans la mesure où le recours à l'Internet et aux réseaux sociaux y augmente constamment. En 2017, dans les pays francophones d'Afrique de l'Ouest, l'utilisation de l'Internet en Côte d'Ivoire était de 44\%, contre respectivement 16\%,12\% et 10\% au Burkina Faso, au Togo et au Niger, ces chiffres étant en augmentation. Les hommes en Afrique subsaharienne sont les principaux utilisateurs de l'Internet, ce qui révèle un écart sur le plan de la dimension de genre dans la mesure où les femmes sont, dans 15\% des cas, moins susceptibles que les hommes de posséder un téléphone mobile et, dans 41\% des cas, plus susceptibles que les hommes d'utiliser l'Internet sur un appareil mobile. ${ }^{5}$ De la même manière, l'utilisation des réseaux sociaux est plus élevée chez les hommes que chez les femmes dans la région, dans la mesure où les femmes représentent entre un et trois utilisateurs d'Instagram et un utilisateur de Facebook sur quatre. ${ }^{6}$

Malgré les proportions relativement faibles de populations des pays à revenus faibles et intermédiaires utilisant l'Internet et les écarts constatés entre les hommes et les femmes, leurs utilisateurs passent environ une heure de plus par jour sur les réseaux sociaux que leurs homologues aux États-Unis. Au niveau mondial, les personnes plus jeunes au sein des pays à revenus faibles et intermédiaires passent deux à trois heures de plus sur les réseaux sociaux que les personnes plus âgées. 'Avec l'usage accru de l'Internet dans ces pays, le volume des conversations sur les réseaux sociaux sera amené à augmenter, élargissant ainsi davantage la disponibilité des données liées aux réseaux sociaux, en particulier parmi les populations plus jeunes.

Les programmes de santé publique peuvent exploiter certaines méthodologies, telles que l'écoute sociale et le suivi social, traditionnellement utilisées dans le domaine du marketing, pour recueillir diverses perspectives sur les connaissances, attitudes et comportements liés à la santé et exprimés par le biais d'interactions en ligne. Ces renseignements peuvent être utilisés conjointement avec d'autres techniques de suivi et d'évaluation pour produire des environnements d'apprentissage riches en données, en vue de concevoir et d'adapter périodiquement des programmes. 


\section{Comment est réalisée l'écoute sociale ?}

L'écoute sociale s’appuie sur les données existantes tirées de sources publiques en ligne. Elle peut être réalisée de manière rétrospective et prospective, permettant ainsi aux parties prenantes dans les domaines des programmes, de la recherche et de l'évaluation en CSC d'accéder à des renseignements pertinents d'une manière rapide et rentable. Parmi les plateformes à travers lesquelles l'écoute sociale peut être réalisée figurent Facebook, Instagram, Reddit, Twitter, YouTube, les blogs, les sites d'informations, parmi tant d'autres.

Les progrès dans le développement des algorithmes d'apprentissage automatique pour l'analyse textuelle ont accru notre capacité à rapidement recueillir et analyser les données issues des réseaux sociaux. Par exemple, l'algorithme d'apprentissage automatique LexRank peut déterminer les messages les plus représentatifs dans une conversation, tandis que le logiciel Crimson Hexagon/Brandwatch Brightview peut classer les messages selon le sujet traité. ${ }^{8}$

En exploitant la force de ce logiciel en matière d'écoute sociale, les analystes peuvent : 1) identifier certains termes essentiels à l'intérieur des conversations et construire des fils de recherche Boolean en utilisant les termes traduits dans toutes les langues requises ; 2) délimiter la période d'écoute ou de suivi souhaitée ; 3) cibler une ou plusieurs plateformes sur les réseaux sociaux; et 4) travailler avec les programmes logiciels d'analyse des réseaux sociaux et les logiciels d'apprentissage automatique.

Concernant le suivi social, les sources sont limitées aux réseaux sociaux employés par le programme CSC pertinent. Les agents de mise en œuvre des programmes peuvent configurer des systèmes de suivi des hashtags (ou mots-dièse) pour évaluer le niveau d'engagement et les réactions aux campagnes ou au contenu ciblé. Par exemple, un programme peut suivre des messages sur la page Facebook ou YouTube d'une campagne, tout en suivant les hashtags liés à la campagne employés sur d'autres plateformes des réseaux sociaux.

Les renseignements obtenus à travers le processus d'écoute sociale sont analysés pour dégager certaines structures dans les messages à échelle réduite afin de déterminer le volume des messages, les pics d'engagement, et les moteurs des conversations, le sentiment derrière elles et les affinités du public, parmi d'autres caractéristiques. Cette analyse peut être similaire à une analyse de données qualitatives à partir d'autres sources. Durant ce processus, tous les messages utilisés dans le cadre de l'analyse ou apparaissant dans des rapports doivent être rendus anonymes en enlevant les noms et les photos de profils. Les plateformes d'écoute sociale ne rendent pas les messages automatiquement anonymes mais cela doit être fait par les analystes, afin de protéger l'identité des sujets ou, dans le contexte d'une recherche, de répondre aux obligations éthiques concernant les sujets humains. Enfin, les renseignements sont partagés avec les gestionnaires de programmes pour déterminer comment utiliser les observations afin d'améliorer le mode de conception et d'adapter le programme.

\section{Comment l'écoute sociale et le suivi social parviennent à orienter les programmes CSC dans les pays francophones d'Afrique de l'Ouest}

Breakthrough RESEARCH collabore avec notre partenaire ressource, M\&C Saatchi, pour appliquer l'écoute sociale et le suivi social à l'orientation des programmes CSC dans les pays francophones d'Afrique de l'Ouest. ${ }^{9}$ Nous fournissons plusieurs exemples tirés de nos expériences dans l'utilisation de ces méthodes à partir des activités Merci Mon Héros appuyées par l'USAID et l'initiative Resilience in the Sahel Enhanced (RISE) II.

\section{La campagne Merci Mon Héros}

Breakthrough ACTION, un programme affilié à Breakthrough RESEARCH, met en œuvre une campagne portant sur les médias de masse et les réseaux sociaux intitulée Merci Mon Héros (MMH) dans neuf pays francophones d'Afrique de l'Ouest (voir Figure 2). Cette campagne est destinée à : 1) encourager les jeunes à faire part de leurs réalités en matière de planification familiale et de santé reproductive (PF/SR); encourager les adultes à surmonter les normes restrictives sur le plan social et en matière de genre pour parler de la PF/SR aux jeunes; et 3) nourrir la discussion entre les jeunes et les adultes pour identifier, aborder et modifier les normes sociales restrictives et éliminer le sentiment de honte et les tabous qui empêchent les jeunes d'accéder aux informations et aux services de PF/SR. ${ }^{10}$

\section{TERMES ESSENTIELS CONCERNANT LE SUIVI DES RÉSEAUX SOCIAUX}

Impressions potentielles totales : Le nombre de personnes qui auraient pu voir une publication donnée sur la base du nombre de followers des personnes impliquées dans ce message.

Portée totale : Le nombre de personnes ayant vu un message au moins une fois. La portée est définie par Facebook comme «le nombre total de personnes ayant vu toute forme de contenu de votre page ou concernant votre page sur leur écran. $»^{15}$

\section{Engagement total/volume des conversations : \\ Le nombre total de fois où les personnes ont été impliquées dans des messages par le biais de réactions, de commentaires, de partages, de retweets, de mentions et de likes.}

Sentiment : La positivité ou négativité globale dégagée par un message. Il reflète le ton des mots clés utilisés dans un message et est mesuré en analysant ces mots clés. 
FIGURE 2. MERCI MON HÉROS

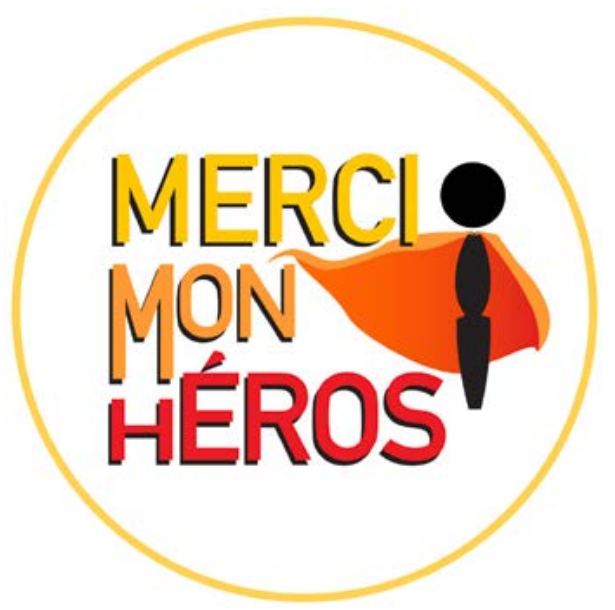

Source: Merci Mon Héros Campaign.

La campagne MMH a été développée par les jeunes à travers une initiative soutenue par Breakthrough ACTION et lancée en ligne en novembre 2019, principalement sur Facebook. Certains messages de la campagne MMH ont également été disséminés à travers d’autres canaux médiatiques traditionnels et plusieurs événements communautaires. Le contenu de la campagne comprenait des vidéos originales de témoignages où apparaissaient des jeunes et des adultes abordant certains sujets comme les premières règles, les premiers rapports sexuels, les premières grossesses, les premières relations et les méthodes de planification familiale.

Breakthrough RESEARCH recourt à l'écoute sociale pour suivre et évaluer la campagne MMH au Burkina Faso, en Côte d'Ivoire, au Niger et au Togo, en conjonction avec d'autres méthodes de recherche. La composante d'écoute sociale aide les parties prenantes à :

- Déterminer les connaissances et les attitudes en rapport à des sujets clés en matière de PF/SR dans les pays cibles.

- Détecter le niveau d'engagement par rapport au contenu de la campagne MMH et formuler des recommandations pour adapter les programmes.

- Reconnaître en quoi les connaissances et attitudes concernant les sujets PF/SR ont évolué au fil du temps.

Un rapport d'écoute sociale couvrant la période s'étalant de novembre 2018 à novembre 2019 a permis de dégager certains thèmes, notamment le rôle de la dimension de genre et de la communication avec le partenaire, à incorporer dans de nouvelles vidéos de la campagne. ${ }^{11}$ La Figure 3 présente le chronogramme de la campagne et le volume des conversations sur Facebook. Les pics dans la courbe illustrent un engagement significatif, ainsi que le contenu ou les événements générant des réponses sur les réseaux sociaux. ${ }^{12}$ Entre le ler novembre 2019 et le 30 juillet 2020, 24023 engagements organiques se sont produits

FIGURE 3. SUIVI DU DEGRÉ D'ENGAGEMENT AVEC LA CAMPAGNE MERCI MON HÉROS

Conversation Volume, Novembre 1-Juin 10, 2020
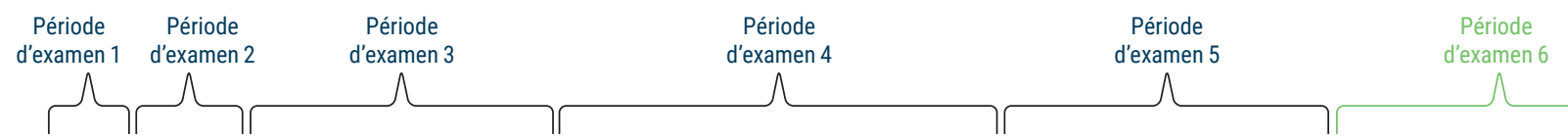

900

800

:...FB Live,

700

600

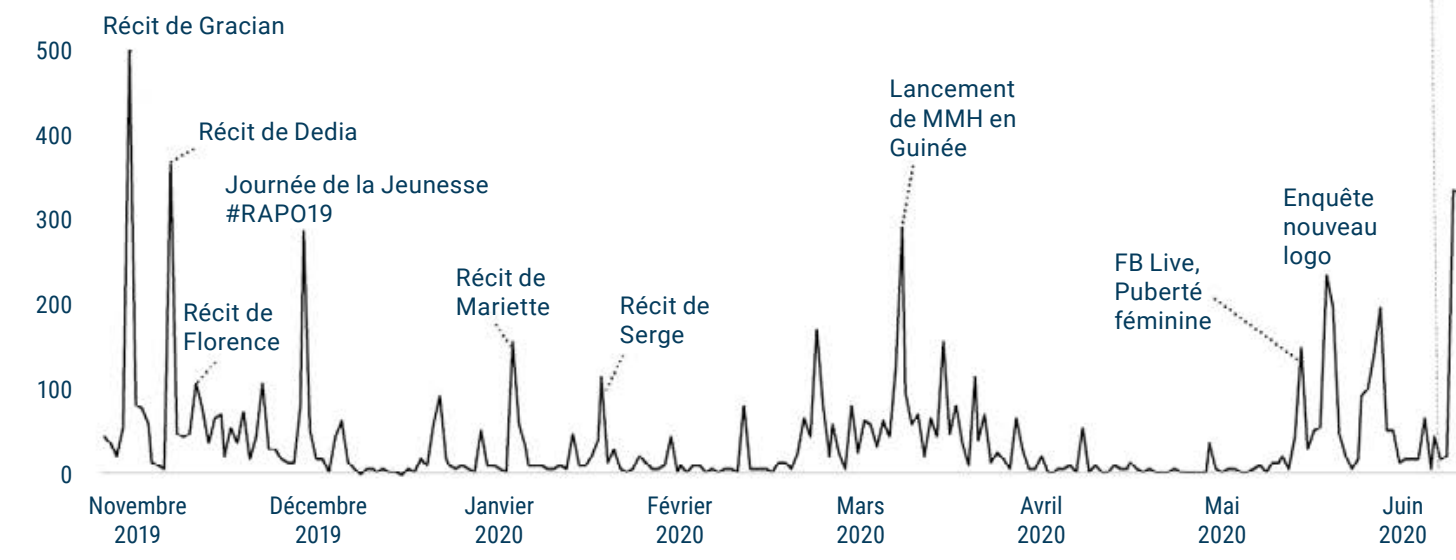

Lancement de $\mathrm{MMH}$ en

Guinée

Cycle menstruel

Source : Breakthrough RESEARCH. 2020. "Merci Mon Héros." Social Listening Monitoring Report \#6. Washington, DC: Population Council. 
à travers les réseaux sociaux et étaient directement liés au contenu de la campagne MMH, notamment les partages, les retweets, les mentions et les commentaires sur Facebook en réponse à des vidéos. Ces rapports ont guidé la stratégie $\mathrm{MMH}$. Par exemple, nous avons observé que la campagne atteignait principalement les jeunes et les jeunes adultes (âgés de 18 à 34 ans), mais pas les adultes plus âgés qui constituent un public d'une importance critique pour inspirer une communication intergénérationnelle permettant de briser certains tabous et d’aborder le sujet de la santé reproductive. Pour y remédier, les chefs de file au sein de la jeunesse ont créé des contenus en ligne spécifiquement orientés vers des adultes plus âgés.

\section{Programme Resilience in the Sahel Enhanced (RISE) II}

Le programme de l'USAID Resilience in the Sahel Enhanced (RISE) II cible les populations chroniquement vulnérables à travers diverses approches pour améliorer certains comportements prioritaires en termes de santé maternelle, néonatale et infantile (SMNI), de planification familiale, de nutrition, et d'accès à l'eau, d'assainissement et d'hygiène. Le programme est mis en œuvre à l'échelle des communautés et au niveau des structures de santé par l'intermédiaire de quatre partenaires d'assistance pour le développement de la sécurité alimentaire et de mécanismes de prestation de services de santé dans des zones sélectionnées au Burkina Faso et au Niger. ${ }^{13}$ Pour appuyer ces partenaires de mise en œuvre, Breakthrough ACTION dispense un renforcement des compétences et une aide technique pour que ces agents améliorent le dispositif de coordination et optimisent la qualité et l'alignement des composantes CSC du programme.

Breakthrough RESEARCH utilise l'écoute sociale et le suivi social pour aider les agents de mise en œuvre de RISE II à déterminer les besoins et priorités parmi les publics cibles au sein des communautés dans le cadre de l'analyse situationnelle et des efforts de planification. Breakthrough RESEARCH enquête sur les réseaux sociaux et les sources des sujets couverts par la presse pour étudier de manière approfondie les conversations en ligne et déterminer les croyances, les attitudes et les normes sociales exprimées à travers les différents sujets ayant trait à la santé, notamment la SMNI, la nutrition, l'accès à l'eau, l'assainissement et l'hygiène.

Les observations préliminaires tirées des conversations sur les réseaux sociaux et de la couverture par la presse nous aident à comprendre comment les jeunes femmes ressentent une pression sociale pour maintenir les pratiques traditionnelles des femmes plus âgées ayant trait à la grossesse et à l'accouchement. Par exemple, comme le montrent les tweets dans la Figure 4, certaines femmes plus âgées peuvent encourager de jeunes mères à éviter de se rendre à l'hôpital ou à adopter certaines pratiques culturelles n'étant pas nécessairement dans l'intérêt de l'enfant et il peut être difficile d'évacuer cette pression.

FIGURE 4. LES POINTS FORTS DE L'ÉCOUTE SOCIALE ET DU SUIVI SOCIAL POUR ORIENTER LES PROGRAMMES CSC DANS LES PAYS FRANCOPHONES D'AFRIQUE DE L'OUEST

On en parle pas assez, des difficultés qu'on les jeunes mères face à
certaines pratiques de leurs mamans et grand mamans. Tu dis "Ne
faites pas ça" et puis la grand mère sort "C'est comme ça qu'on a
fait pour que tu sois ainsi, laisse nous vos affaires de blancs"




\section{Les points forts de l'écoute sociale et du suivi social pour orienter les programmes CSC dans les pays francophones d'Afrique de l'Ouest}

L'écoute sociale et le suivi social présentent bon nombre d'avantages pour les personnes chargées de mettre en œuvre, de suivre ou de réaliser des travaux de recherche sur les programmes cSC, notamment les campagnes de communication.

- L'écoute sociale est un outil extrêmement souple, qui donne aux agents de mise en œuvre et aux chercheurs la capacité d'analyser des données de manière rétrospective ou prospective, sur des intervalles courts ou longs, selon ce qu'autorise le volume de conversations.

- Une production rapide de renseignements peut être réalisée à l'aide du suivi social et de l'écoute sociale de données déjà présentes dans les forums en ligne et les plateformes des réseaux sociaux. Les délais s'écoulant entre le développement du concept de l'étude et la production des renseignements peuvent être réduits à une fraction de ce qu'impliquerait une recherche traditionnelle.

- La technologie évolue très rapidement, et les techniques de suivi social et d'écoute sociale deviendront probablement plus sophistiquées et aboutiront à une meilleure compréhension du ressenti des publics cibles.

\section{Les limites de l'écoute sociale et du suivi social pour orienter les programmes CSC dans les pays francophones d'Afrique de l'Ouest}

Alors que l'écoute sociale et le suivi social offrent certaines possibilités pour recueillir rapidement des informations à partir de conversations en ligne concernant les croyances, les attitudes, les comportements et les normes sociales, plusieurs limites doivent être prises en compte.

- Généraliser les données en ligne représente un risque. En raison des inégalités en matière d'accès à l'Internet et d'utilisation des réseaux sociaux, les données de l'écoute sociale ne peuvent pas être considérées comme représentatives de la population générale. Par conséquent, les observations qui en découlent ne peuvent pas être généralisées. Ce problème est encore aggravé par le fait que certaines considérations liées à la vie privée limitent ce que nous savons des personnes publiant les messages, en particulier sur des plateformes comme Facebook et Instagram.
- Le volume des conversations ne doit pas être considéré comme une unité de mesure précise. Tout d'abord, le fil de recherche est construit pour minimiser les conversations non pertinentes et ne peut pas être efficace à 100\%. D'autre part, certains messages pourraient vraisemblablement chevaucher plusieurs sujets. Néanmoins, ils ne sont attribués qu’à un seul sujet.

- Le sentiment éprouvé par rapport au sujet est difficile à déterminer à l'écrit, malgré la sophistication accrue des outils d'analyse du langage. Cette difficulté est particulièrement avérée quand le sujet en lui-même se manifeste fréquemment dans un langage typiquement considéré comme négatif, tel que « les sévices sexuels ».

- Des bots, des programmes informatiques destinés à interrompre les conversations, peuvent répandre des messages erronés ou prêter à confusion. Selon le sujet abordé, il peut être nécessaire d'examiner certains comptes ou messages pour séparer ceux n'étant pas associés à des utilisateurs réels. ${ }^{14}$

- D'autres biais peuvent être présents sans être pour autant visibles. Les personnes adoptent-elles certaines positions parce qu'elles ont le sentiment de pourvoir en tirer un avantage auprès de leurs amis/followers? Les personnes publiant des messages sur les réseaux sociaux sont-elles plus ou moins susceptibles d'afficher leurs véritables croyances et opinions en ligne plutôt que lorsqu'elles parlent avec quelqu'un en face à face, notamment dans le cadre d'une recherche qualitative plus traditionnelle?

\section{Les réseaux sociaux constituent une nouvelle source d'apprentissage programmatique et de gestion adaptive}

L'utilisation croissante des plateformes des réseaux sociaux donne la possibilité inégalée d'exploiter les conversations en ligne pour suivre et orienter les programmes CSC, en particulier dans les pays avec une utilisation élevée de l'Internet. Les renseignements générés par le biais de l'écoute sociale et du suivi des réseaux sociaux peuvent être utilisés conjointement avec d'autres techniques de suivi et d'évaluation pour produire des environnements d'apprentissage riches en données pour une prise de décision programmatique périodique, et en fin de compte pour améliorer le niveau de performance des programmes. Les agents de mise en œuvre des programmes CSC au niveau mondial et régional, les évaluateurs et les bailleurs de fonds dans les pays prioritaires soutenus par l'USAID doivent envisager d'incorporer l'écoute sociale et le suivi social dans leurs répertoires de méthodes afin de faire part de leurs commentaires et impressions concernant l'apprentissage et la gestion adaptive des programmes. 


\section{References}

1 Simon Kemp. 2020. "Digital 2020: 3.8 billion people use social media," We Are Social, Jan. 30. https://wearesocial.com/ blog/2020/01/digital-2020-3-8-billion-people-use-social-media.

2 En raison de certaines restrictions liées à la protection de la vie privée, WhatsApp n'est pas considéré comme un outil accessible publiquement sur les réseaux sociaux.

3 Tony Train. 2020. "What Is Social Listening, Why It Matters, and 10 Tools to Make It Easier," Hootsuite, March 3, https:// blog.hootsuite.com/social-listening-business/\#whatis

4 — "What Is Social Listening, Why It Matters, and 10 Tools to Make It Easier."

5 GSM Association, Connected Women: The Mobile Gender Gap Report 2019 (2019), https://www.gsma.com/ mobilefordevelopment/wp-content/uploads/2019/02/ GSMA-The-Mobile-Gender-Gap-Report-2019.pdf.

6 NapoleanCat Stats, https://napoleoncat.com/stats/.

7 GlobalWebIndex, Social: Flagship Report 2018, https://www. globalwebindex.com/hubfs/Downloads/Social-H2-2018-report.pdf.

8 Erkan, Güneş, et Dragomir R. Radev. 2004. "LexRank: Graphbased lexical centrality as salience in text summarization," Journal of Artificial Intelligence Research 22: 457-79; pour en savoir plus, consultez https://www.brandwatch.com/.

9 Pour en savoir plus sur le projet Breakthrough RESEARCH, consultez https://breakthroughactionandresearch. org/about/breakthrough-research.

10 Pour en savoir plus sur le projet Breakthrough ACTION, consultez https://breakthroughactionandresearch. org/about/breakthrough-action/.

11 Breakthrough RESEARCH. 2020. "Family planning in West Africa baseline understanding: A social listening report," Breakthrough RESEARCH Technical Report. Washington, DC: Population Council.

12 Breakthrough RESEARCH. 2020. "Merci Mon Héros social listening report \#6: July 2020," Breakthrough RESEARCH Technical Report. Washington DC: Population Council.

13 Dougherty, Leanne et Jessie Pinchoff. 2020. "Evidence to inform an integrated social and behavior change strategy in the Sahel," Breakthrough RESEARCH Literature Review. Washington, DC: Population Council.

14 Broniatowski, David A. et al. 2018. "Weaponized health communication: Twitter bots and Russian trolls amplify the vaccine debate," American Journal of Public Health 108: 1378-84.

15 FACEBOOK for Business, "Reach," https://www. facebook.com/business/help/710746785663278

\section{Remerciements}

Les données présentées dans cette note ont été recueillies et analysées en partenariat avec M\&C Saatchi. Nous tenons à remercier Laura Reichenbach et Paul Hewett du Population Council pour leurs conseils et examens techniques; Nancy Matuszak au Population Reference Bureau pour son soutien éditorial ; Prographics pour la conception du document ; et Lindsay Swisher, Joan Kraft et Alaa Al-Bashir avec l'Agence des États-Unis pour le développement international (USAID), pour leurs retours précieux durant le développement de cette note.

Citation suggérée : Breakthrough RESEARCH. 2020. "Orientation des programmes pour le changement social et de comportement à travers l'écoute sociale et le suivi social, » Breakthrough RESEARCH Note de recherche programmatique. Washington DC : Population Council.

Crédit photo : Merci Mon Héros, John Hopkins Center for Communication Programs

@ 2020 The Population Council. Tous droits réservés.

\section{Breakthrough RESEARCH, Population Council}

4301 Connecticut Ave., NW, Suite 280

Washington, DC 20008

$+1-202-237-9400$

breakthroughactionandresearch.org

BreakthroughRESEARCH@popcouncil.org

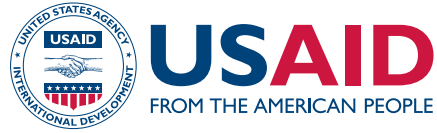

Breakthrough RESEARCH est rendu possible grâce au soutien généreux du peuple américain à travers l'Agence américaine pour le développement international (USAID) en vertu des termes de l'accord de coopération n AID-OAA-A-17-00018. Le contenu de ce document relève de la responsabilité exclusive de Breakthrough RESEARCH et du Population Council et ne reflète pas nécessairement les positions de I'USAID ou du gouvernement des États-Unis.

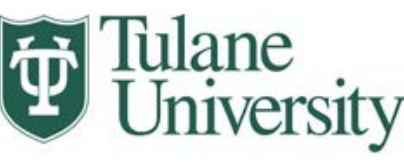

POPULATION COUNCIL

Ideas. Evidence. Impact.

\section{Breakthrough
RESEARCH
FOR SOCIAL \& BEAAVIOR CHANGE \\ Breakthrough
RESEARCH
FOR SOCIAL \& BEAAVIOR CHANGE \\ Breakthrough
RESEARGH
FOR SOCIAL \& Behavior Change}

Cette activité est mise en oeuvre dans le cadre de Breakıthrough RESEARCH. Breakthrough RESEARCH accélère le CSC en effectuant une recherche de pointe et en valorisant des solutions factuelles en vue d'améliorer les programmes de santé et de développement dans le monde. Breakthrough RESEARCH est un consortium dirigé par le Population Council, en partenariat avec Avenir Health, ideas42, l'Institut de santé reproductive de Georgetown University, le Population Reference Bureau et Tulane University. 